\title{
EXPRESSION OF VASCULAR ENDOTHELIAL GROWTH FACTOR DURING NURSE CELL FORMATION IN Trichinella spiralis AND Trichinella pseudospiralis INFECTIONS
}

\author{
KHOSITHARATTANAKOOL P. ${ }^{1}$, MORAKOTE N. ${ }^{1}$, SIRIAUNKGUL S. ${ }^{2}$ AND UPARANUKRAW P. ${ }^{*}$ \\ 1Department of Parasitology, Faculty of Medicine, Chiang Mai University, Chiang Mai 50200, Thailand. \\ 2Department of Pathology, Faculty of Medicine, Chiang Mai University, Chiang Mai 50200, Thailand. \\ *Corresponding Author: Email- puparanu@hotmail.com
}

Received: March 05, 2013; Accepted: March 21, 2013

\begin{abstract}
The two most common Trichinella species causing human disease are T. spiralis and T. pseudospiralis belonging to encapsulated and non-encapsulated clades, respectively. Upon invasion, the larva of $T$. spiralis not only occupies and develops inside the muscle cell but also transforms it into a nurse cell surrounded by the vascular network called circulatory rete. It has been postulated that vascular endothelial growth factor (VEGF) is involved in the rete formation leading to the development and maintenance of nurse cell. This study showed that the expression of VEGF in the nurse cell of $T$. spiralis and $T$. pseudospiralis was detected by in situ hybridization. Basal expression of VEGF was found in uninfected muscle by real-time PCR technique and Western blot analysis. Nevertheless, both methods were not able to demonstrate the increase of VEGF expression above the basal expression in muscles infected with $T$. spiralis or $T$. pseudospiralis. The unexpected results are probably due to the relatively small number of infected nurse cells which could not produce enough positive signals to show significant increase in VEGF expression. It is likely that VEGF plays a role in the formation of circulatory rete and nurse cell in $T$. spiralis infection. However, there has been no experiment to directly validate this hypothesis. This area of study should be further investigated.
\end{abstract}

Keywords- Trichinella, VEGF, Nurse cell, Real-time PCR

Citation: Khositharattanakool P., et al. (2013) Expression of Vascular Endothelial Growth Factor during Nurse Cell Formation in Trichinella spiralis and Trichinella pseudospiralis Infections. International Journal of Parasitology Research, ISSN: 0975-3702 \& E-ISSN: 0975-9182, Volume 5, Issue 1, pp.-111-115.

Copyright: Copyright@2013 Khositharattanakool P., et al. This is an open-access article distributed under the terms of the Creative Commons Attribution License, which permits unrestricted use, distribution and reproduction in any medium, provided the original author and source are credited.

\section{Introduction}

Parasitic nematodes of the genus Trichinella are the etiological agents of the disease called trichinellosis which has a wide host range including birds, reptiles, several mammals as well as human. Trichinella behaves as an intracellular parasite of the striated muscle cell. There are two clades in this genus encompassing encapsulated and non-encapsulated species differentiated by the presence or absence collagen capsule around the nurse cell. $T$. spiralis, the most common encapsulated species, infects the muscle cell and induces remarkable changes that transform the infected muscle cell into a nurse cell that supports the growth and development of the parasite. This process is termed nurse cell formation which involves the loss of muscle proteins, enlargement and division of nurse cell nuclei, mitochondrial damage and formation of collagen [1]. Another obvious feature of the nurse cell in $T$. spiralis infection is the presence of a vascular network called circulatory rete surrounding it [2]. This feature probably corresponds to many observations indicating that larval and nurse cell energy metabolisms are anaerobic [3]. The anaerobic condition could be seen mimicking tissue hypoxia that stimulates angiogenesis through angiogenic factors such as vascular endothelial growth factor (VEGF) in response to hypoxic events [4]. In T. spiralis infection, it was shown that VEGF was expressed in the nurse cell from day 7 up to 8 months and angiogene- sis around the nurse cell began at approximately day 12 and ceased by day 26 [1,5]. Additionally, the in vitro production of VEGF was directly stimulated by $T$. spiralis antigens but not by those of $T$. pseudospiralis [6]. A recent study showed that VEGF protein expression increased in $T$. spiralis-infected muscles at an early phase of infection beginning 7 days after infection and diminished after 3 weeks [7]. The non-encapsulated T. pseudospiralis is also able to form the nurse cell but the collagen capsule, unlike in $T$. spiralis, is poorly developed $[8,9]$. Nevertheless, there has not been any study to demonstrate the presence of angiogenesis around the nurse cell of $T$. pseudospiralis. VEGF has been hypothesized to be involved in the development of the nurse cell-parasite complex in Trichinella infection. However, there has been so far no quantitative study of VEGF transcripts during the development of the nurse cell of both $T$. spiralis and T. pseudospiralis. In the present study, we attempt to determine VEGF expression in both mRNA and protein levels and localize VEGF mRNA transcript during nurse cell formation in $T$. spiralis and T. pseudospiralis infections.

\section{Materials and Methods \\ Parasite Maintenance and Isolation}

The laboratory strain of $T$. spiralis used in this study was isolated from a human in Mae Hong Son, Thailand, in 1986 [10]. The labora- 
tory strain of $T$. pseudospiralis was kindly provided by Professor Yuzo Takahashi, Gifu University, Japan. These parasites were maintained by consecutive passages through ICR mice. T. spiralis and $T$. pseudospiralis infectious first stage larvae (L1) were recovered from muscles of infected mice by digestion with $1 \%$ and $0.8 \%$ pepsin in acidified water, respectively [11]. Digestion was carried out at $37^{\circ} \mathrm{C}$ for $2 \mathrm{hrs}$.or longer by slowly stirring in a hot air incubator (500 ml digestive fluid/mouse). Undigested materials were removed by pouring the digestion through gauze. Larvae were isolated by passing the gauze-filtered digestion through a 200-mesh sieve (Fisher Scientific). The retained larvae were collected from the sieve by washing and suspended in normal saline containing $2 \%(w / v)$ gelatin. Mice were orally inoculated with approximately $250 \mathrm{~L} 1$ by gavaging. Three of infected mice were sacrificed at each time point on days $5,10,15,20,25,30,45$ and 60 of infection. Thigh muscle samples were used for analysis of VEGF transcript levels and protein expression whereas gastrocnemius muscle samples were used for RNA in situ hybridization. The protocol for animal handling was approved by the animal research ethics committee, Faculty of Medicine, Chiang Mai University.

\section{Isolation of Total RNA and Reverse Transcription}

Total RNA was isolated from mouse thigh muscles (uninfected or infected with $T$. spiralis or T. pseudospiralis) or T. spiralis L1 larvae using PureLink'M RNA Mini Kit (Ambion). Genomic DNA was removed from the RNA samples by RNase-free DNase I (Fermentas). Reverse transcription was performed on $0.2-2 \mathrm{mg}$ of total RNA samples using RevertAidTM H Minus Reverse Transcriptase (Fermentas) according to the manufacturer's recommendations.

\section{Preparation of digoxigenin-labeled RNA Probes}

Mouse VEGF DNA was amplified by PCR on the reverse transcription product using a pair of specific primers appended with $\mathrm{T} 7$ and T3 promoter sequences at their 5' end [forward: $5^{\prime}-(T 7)$ GTACCTCCACCATGCCAAGT-3'; reverse: 5'-(T3) AATGCTTTCTCCGC TCTGAA-3']. The primers used for synthesizing $T$. spiralis $18 \mathrm{~S}$ rDNA were 5'-(T7) GATCGCAGCAAGATGAACAA-3' and 5'-(T3) AGACAAATCGCTCCACCAAC-3'. The primers were designed based on the sequences from mouse VEGF gene (GenBank accession number: M95200) and T. spiralis $18 \mathrm{~S}$ ribosomal RNA gene (GenBank accession numbers: U60231 and AY497012), respectively [12]

The sense and antisense RNA probes were generated from VEGF DNA or $T$. spiralis18S rDNA PCR product by in vitro transcription using digoxigenin (DIG)-11-UTP (Roche).

\section{Detection of VEGF mRNA in Muscle Tissue Sections by In-Situ Hybridization (ISH)}

Mouse gastrocnemius muscles infected with $T$. spiralis or T. pseudospiralis at 30 days post-infection were snap-frozen in 2methylbutane chilled with liquid nitrogen and sectioned at $5 \mathrm{~mm}$ thickness using a cryostat. The sections were mounted on the positively charged slides and proceeded to detect the expression of VEGF in the muscle cells by modified ISH method [5,13]. Briefly, the sections were fixed in freshly prepared $4 \%$ paraformaldehyde in PBS for 10 min and followed by washing with DEPC-treated PBS for $5 \mathrm{~min}$ twice. The sections were further prehybridized with hybridization cocktail/ $50 \%$ formamide (Amresco) overnight at RT in a chamber humidified with $5 \mathrm{X}$ SSC. Hybridization was performed by incubating the sections with hybridization cocktail/ $50 \%$ formamide containing $200 \mathrm{ng} / \mathrm{ml}$ DIG-labeled sense or antisense VEGF RNA probe in a chamber humidified with $5 \mathrm{X} \mathrm{SSC} / 50 \%$ formamide overnight at $65^{\circ} \mathrm{C}$. The hybridization mixture was heated to $80^{\circ} \mathrm{C}$ for 5 min, and quickly chilled on ice before applying on the slides. Washing step was done after hybridization by placing the slides in $0.2 \mathrm{X}$ $\mathrm{SSC}$ at $65^{\circ} \mathrm{C}$ for $30 \mathrm{~min}$ twice and at RT for $5 \mathrm{~min}$. Prior to immunological detection, the slides were washed with $100 \mathrm{mM}$ Tris- $\mathrm{HCl} \mathrm{pH}$ $7.5,150 \mathrm{mM} \mathrm{NaCl}$ (buffer 1) for $10 \mathrm{~min}$ and subsequently blocked by covering the sections for 30 min with blocking solution (buffer 1 containing $0.1 \%$ Triton $\mathrm{X}-100$ and $2 \%$ fetal bovine serum). To detect bound RNA probes, the sections were incubated with 1:5,000 antiDIG antibody conjugated with alkaline phosphatase overnight at $4^{\circ}$ C. The sections were then washed with buffer 1 for 10 min three times and further equilibrated for $10 \mathrm{~min}$ with $100 \mathrm{mM}$ Tris- $\mathrm{HCl} \mathrm{pH}$ $9.5,100 \mathrm{mM} \mathrm{NaCl}, 50 \mathrm{mM} \mathrm{MgCl}_{2}$ (buffer 2). The color reaction was developed by nitroblue tetrazolium (NBT)/5-bromo-4-chloro-3indoryl phosphate (BCIP) ready to use (Amresco) in the dark until the desired intensity was reached. Following terminating the color reaction by immersing the slides in $10 \mathrm{mM}$ Tris- $\mathrm{HCl} \mathrm{pH} 8.0,1 \mathrm{mM}$ EDTA (buffer 3) for $5 \mathrm{~min}$, the sections were mounted with a waterbased mounting medium (Kaiser's glycerol gelatin, Merck). The positive reaction was observed as purple color on the tissue under a microscope. Antisense probe of 18S rRNA of the parasite was used as a positive control.

\section{Measurement of VEGF Gene Expression Levels During Nurse Cell Formation by Real-time PCR}

Each reverse transcription product was subjected to real-time PCR in triplicate in $25-\mathrm{ml}$ reaction containing $1 \mathrm{X}$ FastStart Universal SYBR Green Master with ROX (Roche) and $0.3 \mathrm{mM}$ each of forward and reverse primers. The reactions were accomplished using Applied Biosystems 7500 Real-time PCR System instrument according to the thermal cycling profile of $10 \mathrm{~min}$ at $95^{\circ} \mathrm{C}$ followed by 40 cycles of $15 \mathrm{~s}$ at $95^{\circ} \mathrm{C}$ and $60 \mathrm{~s}$ at $60^{\circ} \mathrm{C}$. The primers used in real-time PCR for VEGF were 5'-AATTGAGACCCTGGTGGACA-3' and 5'-CTGCATGGTGATGTTGCTCT-3', and those for GAPDH were 5'-CTCATGACCACAGTCCATGC-3' and 5'-TTCAGCTCTGG GATGACCTT-3'. Following the real-time PCR, the relative expression ratios of VEGF at various time points during the nurse cell formation were calculated based on Ct values of the target (VEGF) and reference (glyceraldehyde-3-phosphate dehydrogenase, GAPDH) genes and their amplification efficiencies using the equation of Pfaffl [14] as shown below.

$$
\text { Ratio }=\frac{\left(E_{\mathrm{VEGF}}\right)^{\left.\Delta \mathrm{Ct} \mathrm{VEGF}_{\mathrm{VE}} \text { (uninfected-infected }\right)}}{\left(\mathrm{E}_{\mathrm{GAPDH}}\right)^{\Delta \mathrm{Ct}_{\mathrm{GAPDH}}(\text { uninfected-infected })}},
$$

Where $E_{V E G F}$ and $E_{G A P D H}$ are the real-time PCR efficiencies of

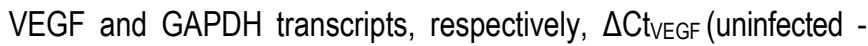
infected) is the difference between the mean of $\mathrm{Ct}(n=3)$ of VEGF gene of uninfected mouse muscles and that of infected mouse muscles, and $\Delta \mathrm{Ct}_{\mathrm{GAPDH}}$ (uninfected - infected) is the difference between the mean of $\mathrm{Ct}(n=3)$ of GAPDH gene of uninfected mouse muscles and that of infected mouse muscles. An experiment was performed to determine the real-time PCR efficiencies and it was found that $E_{V E G F}$ and $E_{G A P D H}$ were 1.88 and 1.81 , respectively. This ratio indicates the relative quantification of the target gene in relation to the reference gene.

\section{Measurement of VEGF Protein Levels by Western Blot Analysis}

Approximately $50 \mathrm{mg}$ of mouse thigh muscle infected with $T$. spiralis or T. pseudospiralis was homogenized in $500 \mathrm{ml}$ protein extraction buffer containing $5 \mathrm{mM}$ Tris-HCl pH 7.4, 1 mM EDTA (pH 8.0), 10\% 
SDS and $1 \mathrm{X}$ mammalian protease inhibitor cocktail (Amresco). To remove insoluble material, the lysate was centrifuged at $10,000 \times \mathrm{g}$ for $10 \mathrm{~min}$. The supernatant was determined protein concentration by the bicinchoninic acid (BCA) protein assay system (Pierce).

Fifty $\mathrm{mg}$ of protein extracts were electrophoresed on $12.5 \%$ SDSPAGE and transferred to PVDF membranes. After blocking with PBS containing $5 \%$ nonfat dry milk and $0.05 \%$ Tween- 20 overnight, the membranes were probed at $4^{\circ} \mathrm{C}$ overnight with $1: 5,000$ rabbit anti-VEGF polyclonal $\mathrm{Ab}$ (Abcam) and 1:20,000 mouse antiGAPDH monoclonal $\mathrm{Ab}$ (Abcam) to detect their respective protein antigens. The membranes were washed with PBS containing $0.05 \%$ Tween-20 for 10 min three times. The membranes were further incubated at RT for $1 \mathrm{Hr}$. with species-appropriate horseradish peroxidase (HRP) conjugated secondary antibody. Following the washing step as before, bound antibody was visualized with 3,3'diaminobenzidine (DAB, Amresco). The intensities of bands were determined using GeneTools program version 4.02 (SynGene). The relative expression of VEGF protein was normalized by dividing the intensities of VEGF bands by those of GAPDH bands.

\section{Results}

\section{VEGF mRNA Expression Levels During Nurse Cell Formation}

The real-time PCR experiment showed that mean relative expressions of VEGF gene during nurse cell formation in $T$. spiralis infection were $0.99,1.33,1.40,1.08,1.35,0.60,0.84,1.01$ and 1.43 for uninfected muscles, muscles at $5,10,15,20,25,30,45$ and 60 days post-infection (DPI), respectively [Fig-1a]. There were no significant differences of VEGF expression among various time points during nurse cell formation of $T$. spiralis (Kruskal-Wallis, $p=0.095$ ).

The results obtained for VEGF expression during nurse cell formation in T. pseudospiralis infection are shown in [Fig-1b]. The mean relative expressions of VEGF gene during nurse cell formation in $T$. pseudospiralis infection were $1.00,1.13,1.94,1.11$, $1.09,1.27,1.03,1.33$ and 1.25 for uninfected muscles, muscles at $5,10,15,20,25,30,45$ and $60 \mathrm{DPI}$, respectively. Like $T$. spiralis, there were no significant differences of VEGF expression among various time points during nurse cell formation of $T$. pseudospiralis (Kruskal-Wallis, $p=0.122$ ).

\section{Localization of VEGF Transcripts by In-Situ Hybridization}

In order to determine the localization of VEGF mRNA in T. spiralisor T. pseudospiralis-infected muscles, RNA in situ hybridization was performed. With the VEGF antisense probe, the purplish stain was observed in T. spiralis-infected muscle cell but not in the larva indicating that VEGF mRNA was localized to the cytoplasm of the nurse cell [Fig-2a]. However, the VEGF antisense probe hybridized not only to the cytoplasm of the nurse cell but also to the larva in $T$. pseudospiralis-infected muscle [Fig-2c]. As expected, the VEGF sense probe did not hybridize to $T$. spiralis-infected muscle cell [Fig2b]. Nevertheless, the sense probe showed weak background hybridization to T. pseudospiralis-infected muscle cell [Fig-2d].

\section{Determination of VEGF Protein Expression During Nurse Cell Formation by Western Blot Analysis}

Western blot analysis was performed to measure VEGF protein expression during nurse cell formation in T. spiralis and T. pseudospiralis infection. Relative expression of VEGF protein was determined by the intensities of VEGF bands normalized by those of GAPDH bands. The 43-kDa VEGF protein and the 37-kDa GAPDH protein in muscle protein extracts from individual mice were recog- nized by anti-VEGF polyclonal antibody and anti-GAPDH monoclonal antibody, respectively [Fig-3]. The results showed that there were no significant differences of the relative VEGF protein levels among uninfected muscles, muscles at 5, 10, 15, 20, 25, 30, 45 and $60 \mathrm{DPI}$ during the nurse cell formation of both $T$. spiralis and $T$. pseudospiralis (Mann-Whitney $U$ test, all $p$ values $>0.05$ ).

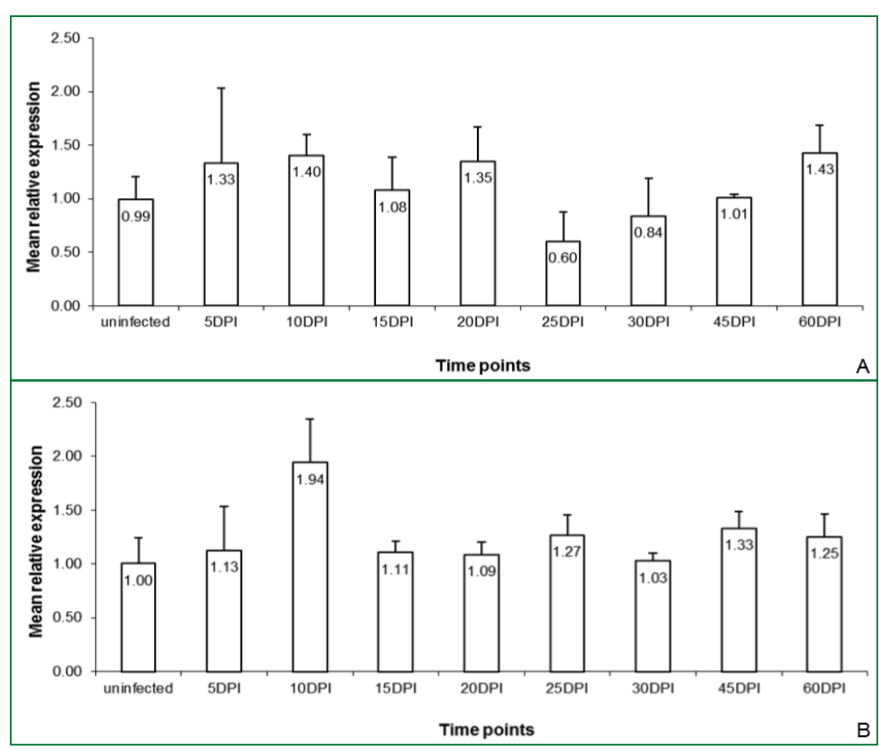

Fig. 1- Mean relative expression of VEGF at various time points during the nurse cell formation of $T$. spiralis $(\mathrm{A})$ and $T$. pseudospiralis $(B)$. Ct values were determined for VEGF gene in triplicate and normalized to $\mathrm{Ct}$ for GAPDH by the method of Pfaffl. Values shown are means of three independent samples. The error bars indicate the standard deviation (SD). Both T. spiralis and T. pseudospiralis infections showed no significant differences during nurse cell formation by non-parametric Kruskal-Wallis test $(p=0.095$ and 0.112 , respectively).

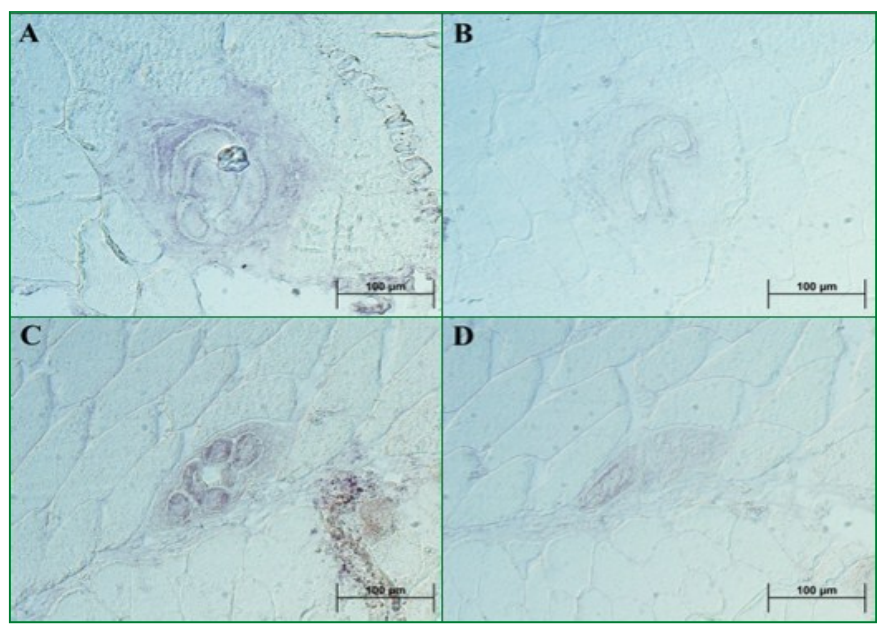

Fig. 2- Detection of mouse VEGF mRNA by in situ hybridization technique. T. spiralis-infected muscle (A and $\mathrm{B}$ ) or T. pseudospiralis -infected muscle ( $C$ and $D) 30$ days post-infection were hybridized with mouse VEGF antisense probe (A and $C$ ) or mouse VEGF sense probe $(B$ and $D)$. The presence of mouse VEGF mRNA is indicated by the purplish stain. Mouse VEGF mRNA was detected in the cytoplasm of the muscle cell infected with $T$. spiralis (A) or $T$. pseudospiralis $(C)$ by the antisense probe. The purplish stain was also observed in T. pseudospiralis larva. Hybridizations with the sense probe were shown in $B$ and $D$ as negative controls. 


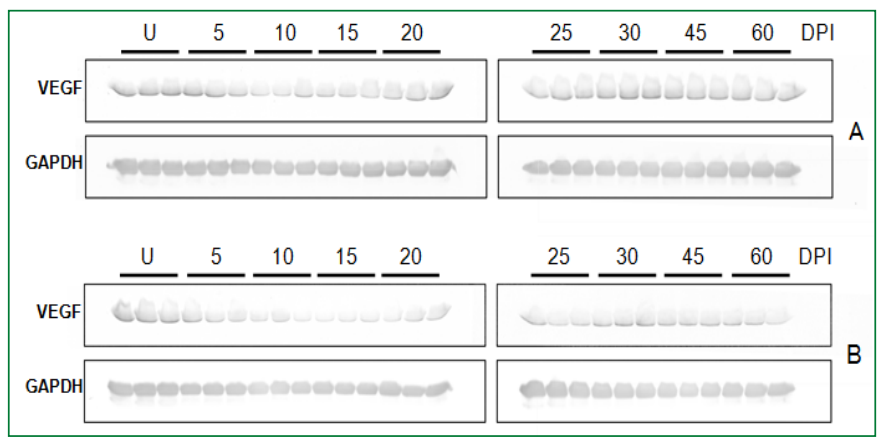

Fig. 3- Western blot analysis of VEGF protein expression in T. spiralis $(\mathrm{A})$ and $T$. pseudospiralis $(\mathrm{B})$ infected muscles. The expressions were analyzed for uninfected muscles $(U)$, muscles at 5,10 , $15,20,25,30,45$ and 60 days post-infection. Muscle extract of three different mice per each time point was recognized with antiVEGF and anti-GAPDH antibodies.

\section{Discussion}

Angiogenesis is a mechanism of adaptive changes to tissue starving and hypoxia due to the limited supply of nutrients and oxygen $[4,15]$. VEGF is one of the most potent regulators of angiogenesis. VEGF binds to its receptors on the surface of endothelial cells in which this incidence induces a variety of signal transductions leading to the formation of new blood vessels [16]. VEGF-induced angiogenesis plays an important role in many conditions such as tumor growth [17,18], psoriasis [19], diabetic retinopathy and retina disorders $[20,21]$ as well as wound repair [22]. Trichinella parasites are very unique in that, upon invasion of the host muscle, their larvae induce not only the transformation of muscle cells into nurse cells but also angiogenesis resulting in the vascular network surrounding the nurse cells [2]. These observations have been studied extensively only in $T$. spiralis infection. The supposed function of the vascular network is to promote the growth of the parasite by supplying nutrients and transporting waste products $[1,23]$. It has been suggested that VEGF might be involved in the vascular formation surrounding the nurse cell since VEGF mRNA was detected in the cytoplasm of the nurse cell from day 7 up to 8 months of $T$. spiralis synchronous infection by RNA in situ hybridization technique [5]. The highest level of VEGF mRNA was detected on $15 \mathrm{DPI}$ in either nurse cell or surrounding inflammatory cells by estimating the reaction intensity. In our study, we confirmed that VEGF mRNA was present in the cytoplasm of the nurse cell of both $T$. spiralis and $T$. pseudospiralis infections but not in the uninfected muscle cells using RNA in situ hybridization technique. Additionally, the more sensitive real-time PCR technique to measure VEGF mRNA expression was performed in this study. The relative quantification by real-time PCR showed that there were no significant differences in the expression of VEGF mRNA during nurse cell formation in either $T$. spiralis or T. pseudospiralis infection. Furthermore, we found that uninfected muscles had a basal expression of VEGF which could be detected by real-time PCR and Western blot analysis. To confirm the above results, another experiment was repeated and the data showed similar patterns of VEGF expression as the previous experiment with insignificant fluctuations of VEGF mRNA levels among various time points during nurse cell formation. The realtime PCR results showing basal VEGF expression in uninfected muscles do not seem to correlate with the in situ hybridization results that did not give any positive signals. This may be explained by the fact that the real-time PCR technique is much more sensitive than the in situ hybridization. Despite its high sensitivity, the realtime PCR technique could not show significant increase in VEGF expression in infected muscles above the basal level whereas VEGF mRNA could be observed in the cytoplasm of nurse cells by in situ hybridization. Presumably, the expression of VEGF in the nurse cells, which constitute a tiny fraction of infected muscle, was not enough to show significant overall elevation by real-time PCR. However, the in situ hybridization, being able to show the localization of positive signals, may better reflect VEGF mRNA expression in the nurse cells indicating that VEGF transcripts were increased in the infected nurse cells. Regarding the expression of VEGF protein in $T$. spiralis-infected muscles during nurse cell formation, a recent study showed that there was an increase of VEGF protein above the basal level in uninfected muscles beginning at day 7 of infection and remained highly expressed until day 42 of infection [7]. In contrast, our study using Western blot analysis showed that VEGF protein expression was not significantly different among various time points (uninfected muscles, muscles at 5, 10, 15, 20, 25, 30, 45 and $60 \mathrm{DPI}$ ) during nurse cell formation in $T$. spiralis and $T$. pseudospiralis infections. Since there was basal expression of VEGF in uninfected muscles in our study, it is possible that VEGF protein expressed in the nurse cells, like its mRNA, was not enough to show significant increase above the basal expression.

The report on different gene expression patterns of the muscles infected with encapsulated parasite, T. spiralis and nonencapsulated parasite, T. pseudospiralis at 23 days after oral infection using cDNA microarray technique demonstrated that there was an up-regulation of VEGF only in T. pseudospiralis- but not in $T$. spiralis-infected muscles (3.09 and 0.98 times of uninfected muscles, respectively) [24]. The microarray data on the expression of VEGF in T. spiralis infection corresponded to our real-time PCR data which showed no difference of VEGF expression between uninfected and infected muscles since both techniques measured the overall expression of VEGF mRNA in the whole muscle. However, our study could not demonstrate the up-regulation of VEGF in $T$. pseudospiralis-infected muscles as in the microarray study.

An in vitro study using crude larval extracts showed that $T$. spiralis but not $T$. pseudospiralis antigens could induce the expression of VEGF mRNA and protein in rat alveolar macrophages [6]. The results indicated that $T$. spiralis antigens were able to stimulate VEGF expression via unknown mechanism. These data support the findings that VEGF mRNA and protein were expressed in infected muscle cells $[5,7]$. These may lead to future studies that could elucidate specific protein(s) responsible for stimulation of VEGF production and as well as its mechanisms.

Up until now there have been only a few publications on VEGF expression in Trichinella infection which still show some conflicting data among those studies and our present study. We therefore still do not completely understand the relationship between VEGF expression and nurse cell formation in Trichinella infection. Although VEGF seemed to increase during nurse cell formation and was likely to induce angiogenesis, it has not been shown that VEGF is directly responsible for the formation of circulatory rete around the nurse cell. Therefore, elucidating the angiogenesis process and VEGF function during nurse cell-parasite complex formation of Trichinella spp. are needed. The experiment that could down regulate the VEGF gene expression (e.g., RNA interference) or interfere with the function of VEGF (e.g., VEGF inhibitors such as chemical inhibitors and anti-VEGF antibody) would directly prove that VEGF 
is indeed involved in the formation of circulatory rete and ultimately the nurse cell-parasite complex. Many recent studies have used anti-VEGF mAbs, which were produced by phage display technology [25], to inhibit functions of VEGF in many diseases such as intestinal adenoma, pituitary adenoma and psoriasis using mouse model [26-28]. An experiment that administers one of the antiVEGF mAbs to Trichinella-infected mice could be tried to see its effects on the larval development and nurse cell formation. Another point of interest is that we have shown for the first time the expression of VEGF mRNA in T. pseudospiralis-infected nurse cell. However, there have not been any studies on the angiogenesis surrounding the nurse cell of any non-encapsulated Trichinella species. It is therefore a good opportunity to study the vascular network surrounding the nurse cell of $T$. pseudospiralis just as has been previously done in $T$. spiralis [2,29].

Conflict of Interests : The authors declare no conflict of interests.

\section{Acknowledgments}

We would like to thank Professor Yuzo Takahashi, Gifu University, Japan and Associate Professor Thidarat Boonmars, Khon Kaen University, Thailand for generously providing T. pseudospiralis. P.K. received a grant from the Thailand Research Fund through the Royal Golden Jubilee Ph.D. program (Grant No. PHD/0101/2548). This study was partially funded by grants from the Faculty of Medicine Endowment Fund, Chiang Mai University.

\section{References}

[1] Despommier D.D. (1998) Parasitol. Today, 14(8), 318-323.

[2] Baruch A.M. and Despommier D.D. (1991) J. Parasitol., 77(1), 99-103.

[3] Stewart G.L. (1983) Biochemistry in Trichinella and Trichinosis, Plenum Press, New York.

[4] Fong G.H. (2008) Angiogenesis, 11(2), 121-140.

[5] Capo V.A., Despommier D.D. and Polvere R.I. (1998) J. Parasitol., 84(2), 209-214.

[6] Shariati F., Perez-Arellano J.L., Lopez-Aban J., Arefi M., Martinez-Fernandez A.R. and Muro A. (2009) Exp. Parasitol., 123 (4), 347-353.

[7] Kang Y.J., Jo J.O., Cho M.K., Yu H.S., Ock M.S. and Cha H.J. (2011) Vet. Parasitol., 181(2-4), 222-228.

[8] Xu D., Wu Z., Nagano I. and Takahashi Y. (1997) Parasitol. Int., 46, 1-5.

[9] Haehling E., Niederkorn J.Y. and Stewart G.L. (1995) Int. J. Parasitol., 25(12), 1393-1400.

[10]Pozio E. and Khamboonruang C. (1989) Trop. Med. Parasitol., 40(1), 73-74.

[11]Crum E.D., Despommier D.D. and McGregor D.D. (1977) Immunolog., 33(6), 787-795.

[12]Claffey K.P., Wilkison W.O. and Spiegelman B.M. (1992) J. Biol. Chem., 267(23), 16317-16322.

[13]Schaeren-Wiemers N. and Gerfin-Moser A. (1993) Histochemistry, 100(6), 431-440.

[14]Pfaffl M.W. (2001) Nucleic Acids Res., 29(9), 2002-2007.

[15]Folkman J. (1971) N. Engl. J. Med., 285(21), 1182-1186.

[16]Olsson A.K., Dimberg A., Kreuger J. and Claesson-Welsh L. (2006) Nat. Rev. Mol. Cell Biol., 7(5), 359-371.
[17]Shweiki D., Itin A., Soffer D. and Keshet E. (1992) Nature, 359 (6398), 843-845.

[18]Plate K.H., Breier G., Weich H.A. and Risau W. (1992) Nature, 359(6398), 846-848.

[19]Detmar M., Brown L.F., Schon M.P., Elicker B.M., Velasco P., Richard L., Fukumura D., Monsky W., Claffey K.P. and Jain R. K. (1998) J. Invest. Dermatol., 111(1), 1-6.

[20]Aiello L.P., Avery R.L., Arrigg P.G., Keyt B.A., Jampel H.D., Shah S.T., Pasquale L.R., Thieme H., Iwamoto M.A., Park J.E. (1994) N. Engl. J. Med., 331(22), 1480-1487.

[21]Adamis A.P., Miller J.W., Bernal M.T., D'Amico D.J., Folkman J., Yeo T.K. and Yeo K.T. (1994) Am. J. Ophthalmol., 118(4), 445-450.

[22]Brown L.F., Yeo K.T., Berse B., Yeo T.K., Senger D.R., Dvorak H.F. and van de W.L. (1992) J. Exp. Med., 176(5), 1375-1379.

[23]Despommier D.D. (1993) J. Parasitol., 79(4), 472-482.

[24]Wu Z., Nagano I. and Takahashi Y. (2008) Parasitol. Int., 57(3), 368-378.

[25]Liang W.C., Wu X., Peale F.V., Lee C.V., Meng Y.G., Gutierrez J., Fu L., Malik A.K., Gerber H.P., Ferrara N. and Fuh G. (2006) J. Biol. Chem., 281(2), 951-961.

[26]Korsisaari N., Ross J., Wu X., Kowanetz M., Pal N., Hall L., Eastham-Anderson J., Forrest W.F., Van B.N., Peale F.V. and Ferrara N. (2008) Clin. Cancer Res., 14(1), 249-258.

[27]Korsisaari N., Kasman I.M., Forrest W.F., Pal N., Bai W., Fuh G., Peale F.V., Smits R. and Ferrara N. (2007) Proc. Natl. Acad. Sci. USA, 104(25), 10625-10630.

[28]Schonthaler H.B., Huggenberger R., Wculek S.K., Detmar M. and Wagner E.F. (2009) Proc. Natl. Acad. Sci. USA, 106(50), 21264-21269.

[29]Humes A.G. and Akers R.P. (1952) Anat. Rec., 114, 103-113. 